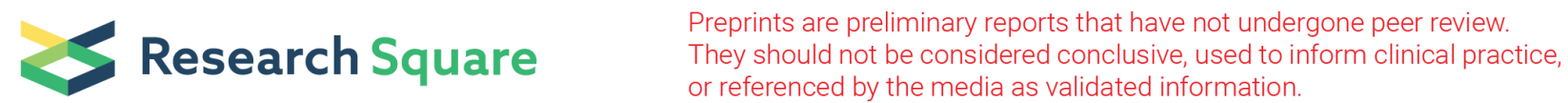

\section{Health Risks and Musculoskeletal Problems of Smartphone-game Elite eSport Players: a Cross- sectional Descriptive Study}

Wing-Kai Lam ( $\sim$ gilbert.lam@connect.polyu.hk)

Shenyang Sport University https://orcid.org/0000-0001-8692-2206

Rui-Tan Liu

Dr. Chen Sport Training and Rehabilitation Research Center, Beijing, 101111, China

Bob Chen

Dr. Chen Sport Training and Rehabilitation Research Center, Beijing, 101111, China

Xin-Zhou Huang

Dr. Chen Sport Training and Rehabilitation Research Center, Beijing, 101111, China

Jie Yi

Dr. Chen Sport Training and Rehabilitation Research Center, Beijing, 101111, China

Duo Wai-Chi Wong

The Hong Kong Polytechnic University Department of Biomedical Engineering

Original Research Article

Keywords: Phone game, sport injury, electronic sport, ergonomic, prolong sitting

Posted Date: February 25th, 2022

DOI: https://doi.org/10.21203/rs.3.rs-1354411/v1

License: (c) (i) This work is licensed under a Creative Commons Attribution 4.0 International License.

Read Full License 


\section{Abstract}

Background: Smartphone eSports athletes sit in the same posture for a prolong period, contributing to significant health risks. This study investigated the health profile, fatigue, pain and complaints, and musculoskeletal problems among full-time smartphone eSports athletes.

Methods: We recruited 50 participants from the ten teams of a top-tier league tournament, with each team for five players. A survey was conducted to evaluate the fatigue pattern, pain level, and complaints. Physical assessment was also conducted by physiotherapists. A descriptive analysis was conducted to evaluate the health profile (BMI, fat ratio), fatigue, number of complaints and musculoskeletal problems. The associations of career duration with $\mathrm{BMI}$, fat ratio, and the total number of confirmed injuries were then performed using a Spearman's rank correlation test.

Results: About $46 \%$ and $44 \%$ of participants felt tired, while $34 \%$ and $58 \%$ experienced eyestrain frequently and occasionally. More than $30 \%$ of the participants reported headache, neck pain, trigger finger and rhinitis. Physiotherapists confirmed head-and-trunk injuries on $90 \%$ of the participants, with a quarter of them suffering at least one of these four pains. Longer career duration of eSports may be associated with a reduction in $\mathrm{BMI}(\mathrm{r}=-0.272, p=0.056)$. The duration of career has no significant association with smoking habit $(p=0.666)$, alcohol habit $(p=0.655)$, coffee habit $(p=0.946)$, rounds of game that can maintain concentration $(p=0.253)$, ease of eyestrain $(p=0.569)$, tiredness $(p=0.510)$, dizziness $(p=0.071)$, and leg numbness $(p=0.318)$.

Conclusion: The findings of this study stressed the significance of eSports injuries and implicated preventive measures for both athletes and phubbers.

\section{Key Points}

- More than $90 \%$ of participants felt tired and experienced eyestrain after prolong eSports gaming.

- More than $30 \%$ of the participants reported headache, neck pain, trigger finger and rhinitis.

- A longer career duration of eSports may potentially be associated with a small decrease in BMI

\section{Introduction}

Electronic Sports (eSports) is a form of competition that players or teams battle in a virtual multiplayer video game through electronic interfaces (computer or smartphone), particularly in a professional context. eSports have been accepted as a medal event in Asian Games and Olympic Games in 2022 and 2024, respectively [1]. Over 2.5 billion players engaged in virtual and competitive electronic gaming worldwide [2] and over 50 university gaming teams in the past three years [3]. In China, about 560 million people, equivalent to $70 \%$ of the country online population, have played computer and smartphone games regularly, which accounts for US\$ 11.1 billion eSports streams in 2016 [4]. Previous research 
estimated total global revenue of US\$152 billion in 2019 and forecasted over US\$257 billion for eSport industry by 2025 [2].

Unlike traditional sports that promote physical fitness, full-time eSport athletes sit 5.5 to more than 10 hours a day during their regular game training for superior eye-hand coordination, fast reaction time, and rapid decision making $[2,3,5,6]$. The high training intensity under specific postural behaviour and workplace environment may induce health risks similar to sedentary lifestyles [5, 7]. Previous studies reported that 6 to 8 hours of sitting may increase all-cause mortality, cardiovascular disease mortality [8], and orthopaedic injuries due to poor postures [3.9.10]. Understanding the pattern, frequency, and distributions of eSport injury could facilitate better health management for players, especially towards eye strain, repetitive hand injury, and other musculoskeletal problems.

ESports Injury and health investigations have focused on computer-based eSports, but are lacking in smartphone-based eSports gaming [2, 3]. Literature indicated that eSports might lead to game addiction affecting other daily activities and lower working efficiency [4]. ESports were also linked to several mental health problems, such as anxiety disorder, depression, aggressive behaviour, and suicidal ideation $[2,4$, $11,12]$. While professional eSport athletes stay sitting and staring on screens, professional eSport athletes often complains of eye fatigues and physical ailments in trunk, upper limb and lower limb such as back strains, carpel tunnel, trigger finger, lower crossed syndrome, and deep vein thrombosis $[2,3,9]$. In fact, smartphone-based gamers have different playing postures, environment, and player-environment interactions from computer-based gamers, such as differences in screen sizes, viewing angle, finger, hand, and arm motion, etc. Therefore, they may have different postural adaptation, muscle fatigue/strain, and repetitive injury patterns after prolonged and high-intensity training.

In particular, health risks of high-intensity smartphone gaming or usage are potential public health burdens that may not be confined to professional players. With the popularity of high-performance mobile phones, mobile phone games, and speedy $5 \mathrm{G}$ network, adolescents and adults spent their days on smartphones for communication, entertainment, and work with a high incidence of related orthopaedic injuries $[13,14]$. The investigation on eSport competitors could provide insights on the underlying health or musculoskeletal problems that provide further insights on the education for and prevention of injuries. Hence, the objective of this study was to understand the health risks and complaints among full-time smartphone eSports elite players of different career durations.

\section{Methods}

\subsection{Participants}

We recruited 50 male smartphone eSports elite athletes from the ten teams of a top-tier league tournament, [Onmyoji Arena Pro League (OPL) hosted in 2021 spring in Shanghai], with each team with five players. It was a 10-team tournament with a 5-on-5 game competition. The basic demographic information is available in Table 1. The study was approved by the Institutional Review Broad. All participants signed an informed consent form prior to the commencement of the study. 
Table 1

Demographic information $(\mathrm{N}=50)$

\begin{tabular}{|ll|}
\hline Variables & Value \\
\hline Age, Mean \pm SD (year) & $20.0 \pm 1.67$ \\
\hline Age, Range (year) & $18-24$ \\
\hline Body Height, Mean \pm SD $(\mathrm{kg})$ & $1.76 \pm 0.06$ \\
\hline Body Weight, Mean \pm SD (m) & $70.82 \pm 17.6$ \\
\hline BMI, Mean \pm SD $\left(\mathrm{kg} / \mathrm{m}^{2}\right)$ & $22.86 \pm 5.11$ \\
\hline Fat Ratio, Mean \pm SD $(\%)$ & $18.69 \pm 8.10$ \\
\hline Career duration, Median (months) & 6 \\
\hline Career duration, IRQ (months) & $3-11$ \\
\hline Individuals with habits (n, \%): & $11,22 \%$ \\
\hline$\cdot$ Smoking & $19,38 \%$ \\
\hline$\cdot$ Alcohol & $28,56 \%$ \\
\hline$\cdot$ Coffee & \\
\hline SD: standard deviation; BMl: body mass index; IRQ: Interquartile range \\
\hline
\end{tabular}

\subsection{Experiment protocol}

The study was conducted during the off-season period (i.e., Sep 2021). We have conducted a survey and interview with the participants. Demographics, habits (smoking, alcohol, coffee), and career information were collected. Fat ratio (body fat percentage) was measured when the participants standing upright and holding the handrails of the bioelectrical impedance body fat measurement device (Body fat monitor HBF-302, OmRon, Japan). During the interview, the participants reported their health complaints: dry eyes, headache, dizziness, sleep disturbance, anxiety, shoulder pain, periscapular pain, hemorrhoids, gastroenteritis, angina, and rhinitis. Moreover, the fatigue features were assessed by a supervised survey approach $^{15}$ and the questions in the survey are provided in Table 2. 
Table 2

Items in the supervised questionnaire

\begin{tabular}{|c|c|c|}
\hline Items & Questions & Options \\
\hline \multirow[t]{3}{*}{ a } & \multirow{3}{*}{$\begin{array}{l}\text { How many sets of game competitions at most can you maintain } \\
\text { concentration? }\end{array}$} & - 1 set \\
\hline & & - 2 set \\
\hline & & - 3 set \\
\hline \multirow[t]{4}{*}{ b } & \multirow[t]{4}{*}{ When do you easily feel tired in the day? } & - Morning \\
\hline & & - Afternoon \\
\hline & & - Evening \\
\hline & & • Mid-night \\
\hline \multirow[t]{3}{*}{ c } & \multirow[t]{3}{*}{ How often do you get tired after prolong gameplay? } & • Never \\
\hline & & - Occasionally \\
\hline & & - Frequently \\
\hline \multirow[t]{3}{*}{ d } & \multirow[t]{3}{*}{ How often do you get eyestrain after prolong gameplay? } & • Never \\
\hline & & - Occasionally \\
\hline & & • Frequently \\
\hline \multirow[t]{3}{*}{ e } & \multirow{3}{*}{$\begin{array}{l}\text { How often do you feel dizziness (i.e., feel like a lack of blood supply to the } \\
\text { brain) after prolong gameplay? }\end{array}$} & • Never \\
\hline & & - Occasionally \\
\hline & & • Frequently \\
\hline \multirow[t]{3}{*}{ f } & \multirow[t]{3}{*}{ How long does it take to have leg numbness in a training or competition? } & • Never \\
\hline & & - About 4-h \\
\hline & & - About 8-h \\
\hline \multirow[t]{3}{*}{ g } & \multirow[t]{3}{*}{ Will you decide to relax yourself after training? } & • Never \\
\hline & & - Occasionally \\
\hline & & - Frequently \\
\hline
\end{tabular}

Physical musculoskeletal examinations were performed by three registered physiotherapists over the neck, shoulders, elbows, wrists/hands/fingers, upper back, lower back, hips/thighs, and knees. The number of confirmed injuries on the upper extremity, trunk-and-head, and lower extremity regions were recorded by the physiotherapists. A pain scale (10-point Likert scale) was used to indicate and categorize the level of subjective pain for the confirmed injured regions if any [16], 0 point: no pain; 1 to 3 points: 
mild; 4 to 6 points: moderate and affecting normal functionality; and 7 to 9 points: severe and need to seek medication.

\subsection{Data analysis}

A descriptive analysis was conducted to evaluate the health profile (BMI and fat ratio), fatigue features, number of self-reported complaints and musculoskeletal problems among the participants. For the musculoskeletal problems, the level of pain was also presented. Then, we analyzed the association of career duration with BMI, fat ratio, and the total number of confirmed injuries using a Spearman's rank correlation test.

Afterward, we categorized the participants into three groups based on their career duration: 1) < 6 months; 2) 7-12 months; and 3) over 11 months. The associations between the categorized career duration, habits (smoking, alcohol, and coffee), and selected fatigue features, including concentration tolerance, ease of eyestrain, tiredness, dizziness, and leg numbness, were evaluated using the Chi-square test. If the data assumption for Chi-square was violated, the likelihood ratio would be implemented. The significance level was set at $p<0.05$ for all analyses. All statistical analyses were performed using the SPSS 26.0 software (IBM, Armonk, NY, USA).

\section{Results}

For the fatigue features, as shown in Fig. 1,96\% of elite athletes could maintain attentional concentration for two or three sets of the game. Tiredness was usually onset in the afternoon (48\%) and evening (28\%). Approximately $46 \%$ and $34 \%$ of the athletes felt tiredness and eyestrain frequently, and another $44 \%$ and $58 \%$ of them felt occasionally. More than one-tenth of the athletes reported frequent dizziness, and $68 \%$ of them had occasional dizziness. More than a quarter of participants experienced leg numbness after 4 hours of training. More than one-fifth of the athletes headed for relaxation after training or competition.

Figure 2 summarizes the self-reported health complaints. More than $30 \%$ of participants suffered from headache cervical spondylosis, trigger fingers (finger tenosynovitis), and rhinitis. For the physiotherapist assessment, only one participant (2\%) was free from injury. $46 \%, 90 \%$, and $12 \%$ of the participants confirmed with at least one upper limb, head-and-trunk, and lower limb injuries, respectively. For the headand-trunk injuries, $78 \%$ of the participants had injuries in at least two regions, and $28 \%$ had injuries in at least four regions. For the pain scale in Table 3, neck pain was the most prevalent complaint, followed by finger pain and headache. They contributed $40 \%, 38 \%$, and $32 \%$ of the cases, respectively. About one-fifth and one-quarter of the participants suffered from low back pain and upper pain. Although the majority of the pain was mild, there were 4 cases of moderate finger pain and 3 cases of moderate headache. 
Table 3

Level of pain of specific regions graded by a 10-point Likert scale (Nil: 0, Mild: 1 to 3, Moderate: 4 to 6 , Severe: 7 to 9 )

\begin{tabular}{|c|c|c|c|c|c|c|c|c|c|c|c|c|c|c|}
\hline \multirow[b]{2}{*}{ Pain location } & \multirow[b]{2}{*}{ Case } & \multirow[b]{2}{*}{$(\%)$} & \multicolumn{4}{|c|}{ Mild } & \multicolumn{4}{|c|}{ Moderate } & \multicolumn{4}{|c|}{ Severe } \\
\hline & & & 1 & 2 & 3 & Total & 4 & 5 & 6 & Total & 7 & 8 & c & Total \\
\hline Headache & 16 & $(32 \%)$ & - & 7 & 6 & 13 & 2 & 1 & - & 3 & - & - & - & 0 \\
\hline Eyestrain & 9 & $(18 \%)$ & 1 & 8 & - & 9 & - & - & - & 0 & - & - & - & 0 \\
\hline Neck pain & 20 & $(40 \%)$ & 1 & 11 & 8 & 20 & - & - & - & 0 & - & - & - & 0 \\
\hline Shoulder pain & 8 & $(16 \%)$ & 2 & 5 & - & 7 & 1 & - & - & 1 & - & - & - & 0 \\
\hline $\begin{array}{l}\text { Upper back } \\
\text { pain }\end{array}$ & 12 & $(24 \%)$ & - & 3 & 9 & 12 & - & - & - & 0 & - & - & - & 0 \\
\hline Finger pain & 19 & $(38 \%)$ & 1 & 12 & 2 & 15 & 4 & - & - & 4 & - & - & - & 0 \\
\hline Wrist pain & 4 & $(8 \%)$ & - & 2 & 2 & 4 & - & - & - & 0 & - & - & - & 0 \\
\hline $\begin{array}{l}\text { Lower back } \\
\text { pain }\end{array}$ & 10 & $(20 \%)$ & 1 & 2 & 5 & 8 & 2 & - & - & 2 & - & - & - & 0 \\
\hline Knee pain & 3 & $(6 \%)$ & - & 2 & 1 & 3 & - & - & - & 0 & - & - & - & 0 \\
\hline
\end{tabular}

The career duration had no significant association with the fat ratio $(r=-0.229, p=0.110)$, and total number of confirmed injuries $(r=-0.155, p=0.284)$. However, there was a small negative correlation between career duration and BMI with marginal significant difference $(r=-0.272, p=0.056)$. In addition, career duration did not demonstrate significant association with smoking habit $(p=0.666)$, alcohol habit ( $p=0.655)$, coffee habit $(p=0.946)$, rounds of game that can maintain concentration $(p=0.253)$, ease of eyestrain $(p=0.569)$, tiredness $(p=0.510)$, dizziness $(p=0.071)$, and leg numbness $(p=0.318)$ in the likelihood ratio tests.

\section{Discussion}

Smartphone games and competitive gaming are becoming increasingly recreational and Olympics activities with blooming popularity worldwide $[2,3,17]$. However, a number of occupational factors, including prolonged screen time, repetitive movements, poor posture, and sedentary behaviour have been identified to increase the potential risk for the development of chronic diseases and possibly all-cause mortality $[8,18]$. This study aimed to examine the training behaviour, fatigue profile, and location of pain/discomfort among professional smartphone athletes. The present findings revealed that most professional esport athletes would induce physical fatigues and dry eyes after half-day (4-hour) and fullday (8-hour) training. Furthermore, similar to previous demographic studies, eSport athletes are required to sit with the same position for 5.5 to $10 \mathrm{~h} /$ day $[3,5]$ or up to $14 \mathrm{~h} /$ day [2], leading to a sedentary lifestyle 
with an increased chance of fatigue and chronic injury as well as increased all-cause mortality $[8,18]$. The physical fatigue may be due to poor workplace practices (prolong posture, repetitive movement), competition stress, and burnout; while eye fatigues could also be related to prolonged visual attention to the screen and poor lighting environment [3].

Fatigue is a common symptom of many medical conditions, from mild-to-severe. It can cause various physical, mental, and emotional symptoms, including headaches/dizziness, sore muscles, muscle imbalance, poor concentration, impaired decision-making, and hand-to-eye coordination [19]. Our data indicated that nearly half of the athletes were actively performed regular relaxation/recovery after eSport training and competition, which agrees with the previous study showing a $29.2 \%$ and $34.2 \%$ of the videogame athletes had relaxation/recovery and physical fitness sessions, respectively [17]. Therefore, a regular fatigue monitoring and management session were recommended to pertain to the athlete's maximal physical performance, injury, and illness risk, especially after half-day and full-day training.

Over $30 \%$ of the eSport athletes reported cervical spondylosis, headache/dizziness, phalanx tenosynovitis, and rhinitis. They could be related to the specific ergonomic and activity demand. In typical smartphone game athletes, maintaining posture with the prolonged inferior viewing angle and hand-held smartphone position would induce head flexion, repeated or sustained wrist bending, and repeated twisting or pushing thumb motions [15]. Athletes may develop muscle imbalance between agonist and antagonist muscles and thus poor balance and muscle soreness. Additionally, our results indicated the highest prevalence rates of professional smartphone gaming athletes were neck (40\%), finger (38\%), and headache (32\%), which were slightly different from the rates from the video gaming athletes [Neck (42\%), back $(42 \%)$, wrist $(36 \%)$, and hand (32\%)][3] and from the sedimentary office workers [lower back (72\%) and neck (55.2\%)] [20]. The differences in prevalence rates and locations could be due to the unique workplace environment and task intensity in smartphone eSport, which affect fine-tuning of postural control and postural adaptation ${ }^{21}$ and thereby develop chronic injuries [2.9].

Interestingly, our results indicated that while career duration had no significant association with the fat ratio and total confirmed injury, but it showed a marginal negative association with BMI instead, which did not support our original hypothesis. It is expected that prolong sitting time in smartphone game training could be similar to sedentary lifestyles [5], which could lead to physical inactive that is related to an increased $\mathrm{BMI}$ and fat ratio and health risks such as hypertension and diabetes. One plausible explanation is due to a relatively short lifespan of the career in professional athletes, officially start at 18 and normally retire at 24 years old. Young sport athletes could be more likelihood to attain superior eyehand coordination (500 to 600 action moves/min) and rapid decision making and reaction times $[3,6]$. Another explanation is due to the movement intensity of eSport is as high as combat sports and might develop only mild injuries, which may not be easily recurrence over years as other traditional sports such as basketball, soccer and badminton. To our best knowledge, this is the first work that shows the fatigue and injury profiles of professional smartphone eSport athletes. 
Some limitations should be considered when interpreting our results. First, only a single group of elite male smartphone athletes from Mainland China top teams was recruited in this study. The results may not be generalized to lower-level game players as well as recreational smartphone gamers. Second, only the self-report and orthopaedic examination report were provided. Other comprehensive physiological and performance indicators (e.g., eye-hand coordination, decision-making capacity, visual attention) should also be considered to facilitate the identification of talented players. Third, the small sample size in this study had low frequency distribution and therefore we used likelihood ratio instead of Chi-square with less statistical powerful. Forth, the fatigue and injury profiles are rather game-content specific, which may affect the decision-making and motor skill control (thumb vs. index finger) to attain the best results in a game. Although all the participants were trained with the same game-content in this season, more gamecontent should also be investigated to determine how game-content would influence the health injury. This can be served as the game design guidelines (e.g., length of each game set, use of control setting) for game designers and policy makers.

\section{Conclusion}

We found a high prevalence of headache, neck pain, and finger pain and a high occurrence of head-andtrunk injuries among smartphone elite eSports athletes, despite that no significant association was found with the career duration. The future study shall consider investigating the influence of different intensities and exposures of smartphone games and direct preventive measures for phubbers.

\section{Declarations}

\section{Ethics Approval and Consent to Participate}

The authors was approved by the institutional ethics committee of the Hong Kong Polytechnic University. The study was performed in accordance with the 1964 Declaration of Helsinki. All participants was provided informed consent prior to participation.

\section{Consent for Publication}

Not applicable.

\section{Availability of data and materials}

The datasets used and/or analyzed during the current study are available from the corresponding author on reasonable request.

\section{Completing Interests}

RTL, BC, X-ZH and JY work for Dr. Chen Sport Training and Rehabilitation Research Center, which provide orthopaedic examination services to all eSport teams in China. Other authors declare that they have no conflicts of interest relevant to the content of this manuscript. 


\section{Funding}

No financial support was provided for this study.

\section{Authors' contributions}

All authors contributed substantially to the preparation of this manuscript. RTL, BC, X-ZH and JY were responsible for protocol design. RTL, X-ZH and JY were responsible for eSport physical assessment and data collection. W-KL, RTL and DW-CW were responsible data analysis. All authors interpreted the data. $\mathrm{W}-\mathrm{KL}$ and DW-CW wrote the first draft. All authors revised and finalized the manuscript.

\section{Acknowledgements}

We would like to give thank the coaches and managers of the eSport teams to allow for orthopaedics assessment and data collection.

\section{References}

1. Wattanapisit A, Wattanapisit S, Wongsiri S. Public health perspectives on eSports. Pub Health Rep. 2020;135(3):295-8; https://doi.org/10.1177/0033354920912718

2. Truong P, Truong L, Le T, Kuklova K. Orthopedic injuries from video games: A literature review and implications for the future. Int Arch Orthop Surg. 2020;3(2):020; https://doi.org/10.23937/26434016/1710020

3. DiFrancisco-Donoghue J, Balentine J, Schmidt G, Zwibel H. Managing the health of the eSport athlete: an integrated health management model. BMJ Open Sport Exer Med. 2019;5:e000467; https://doi.org/10.1136/bmjsem-2018-000467

4. Chung T, Sum S, Chan M, Lai E, Cheng N. Will esports result in a higher prevalence of problematic gaming? A review of the global situation. J Behav Additions; 2019;8(3):384-94; https://doi.org/10.1556/2006.8.2019.46.

5. Emara AK, Ng MK, Cruickshank JA, Kampert MW, Piuzzi NS, Schaffer JL, King D. Gamer's health guide: Optimizing performance, recognizing hazards, and promoting wellness in Esport; Cur Sport Med Rep. 2020;19(12):537-45; https://doi.org/10.1249/JSR.0000000000000787.

6. Lewis JM, Trinh P, Kirsh D. A corpus analysis of strategy video game play in Starcraft: Brood War. Proceedings of the $33^{\text {rd }}$ annual conference of the cognitive science society 2011.

7. Yin K, Zi Y, Zhuang W, Gao Y, Tong Y, Song L, Liu Y. Linking Esports to health risks and benefits: Current knowledge and future research needs. J Sport Health Sci. 2020;9:485-8; https://doi.org/10.1016/j.jshs.2020.04.006.

8. Patterson R, McNamara E, Taino M, de Sa TH, Smith AD, Sharp SJ, et al. Sedentary behaviour and risk of all-cause, cardiovascular and cancer mortality, and incident type 2 diabetes: a systematic review and dose response meta-analysis. Eur J Epidemiol. 2018;33(9):811-29; https://doi.org/10.1007/s10654-018-0380-1. 
9. McGee C, Ho K. Tendinopathies in video gaming and Esports. Front Sport Active Living. 2021;3:689371; https://doi.org/10.3389/fspor.2021.689371.

10. Pourmand A, Lombardi K, Kuhl E, O'Connell F. Videogame-related illness and injury: A review of the literature and predicrtions for Pokemon Go! Game Health J: Res Dev Clin Appl. 2017;6(1):1-10; https://doi.org/10.1089/g4h.2016.0090.

11. Park S, Jeon HJ, Bae JN, Seong SJ, Hong JP. Prevalence and psychiatric comorbidities of internet addition in a nationwide sample of Korean adults. Psychiatry Investig. 2017;14(6):879-82; https://doi.org/10.4306/pi.2017.14.6.879

12. Messias E, Castro J, Saini A, Usman M, Peeples D. Sadness, suicide, and their association with video game and internet overuse among teens: Results from the youth risk behavior survey 2007 and 2009 . Suicide Life-Threatening Behav. 2011;41(3):307-15; https://doi.org/10.1111/j.1943278X.2011.00030.x.

13. AlAbdulwahab SS, Kachanathu SJ, AIMotairi MS. Smartphone use addiction can cause neck disability. Musculoskel Care. 2017;2017:1-3.

14. Betsch M, Kalbhen K, Michalik R, Schenker H, Gatz M, Quack V, Siebers H, Wild M, Migliorini F. The influence of smartphone use on spinal posture - A laboratory study. Gait Posture. 2021;85:298-303; https://doi.org/10.1016/j.gaitpost.2021.02.018

15. Ma T, Song L, Ning S, Wang H, Zhang G, Wu Z. Relationship between the incidence of de Quervain's disease among teenagers and mobile gaming. Int Orthop. 2019;43(11):2587-93; https://doi.org/10.1007/s00264-019-04389-9

16. Ferreira-Valente MA, Pais-Ribeiro JL, Jensen MP. Validity of four pain intensity rating scales. Pain. 2011;152:2399-404; https://doi.org/10.1016/j.pain.2011.07.005

17. Rudolf K, Bickmann P, Frobose I, Tholl C, Wechsler K, Grieben C. Demographics and health behaviour of video game and eSport players in Germany: The eSports study 2019. International J Env Res Pub Health. 2020;17:1870; https://doi.org/10.3390/ijerph17061870

18. Biswas A, Oh PI, Faulkner GE, Bajaj RR, Silver MA, Mitchell MS, Alter DA. Sedentary time and its association with risk for disease incidence, mortality, and hospitalization in adults: A systematic review and meta-analysis. Ann Int Med. 2015;162:123-132; https://doi.org/10.7326/M14-1651

19. Thorpe RT, Atkinson G, Drust B, Gregson W. Monitoring fatigue status in elite team - sport athletes: Implications for practice. Int J Sport Physiol Perf. 2017;12(2):s227-s234; https://doi.org/10.1123/ijspp.2016-0434.

20. Mohammadipour F, Pourranjbar M, Naderi S, Rafie F. Work-related musculoskeletal disorders in Iranian office workers: Prevalence and risk factors. J Med Life. 2018;11(4):328-333; https://doi.org/10.25122/jml-2018-0054

21. Frost HM. Wolff's Law and bone's structural adaptations to mechanical usage: an overview for clinicians. Angle Orthodontist. 1994;64(3):175-188; https://doi.org/10.1043/00033219(1994)064<0175:WLABSA>2.0.C0;2. 


\section{Figures}

a. How many sets at most can you maintain concentration

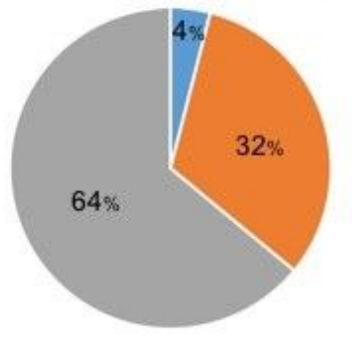

c. How often do you get tired after prolong gameplay

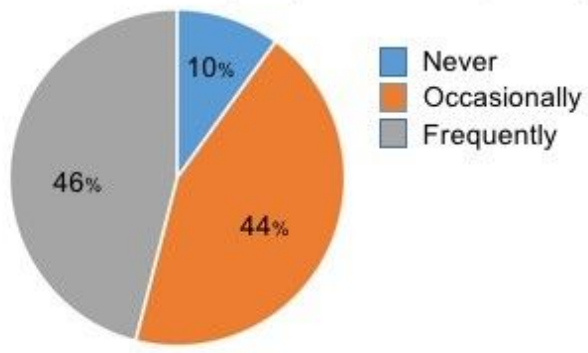

e. How often do you feel dizziness after prolong gameplay

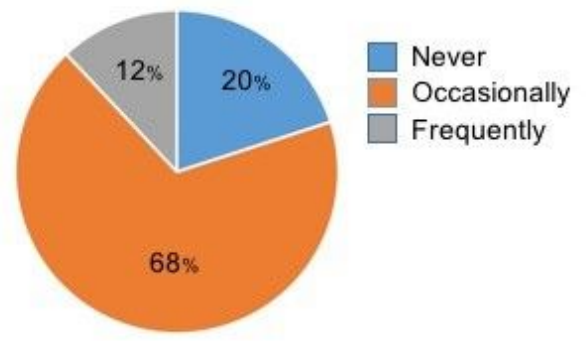

g. Will you decide to relax yourself after training

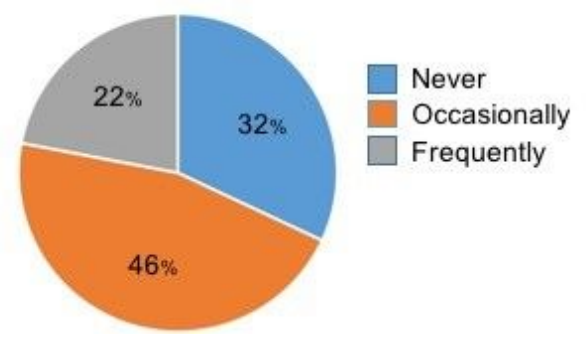

b. What do you easily feel tired in the day

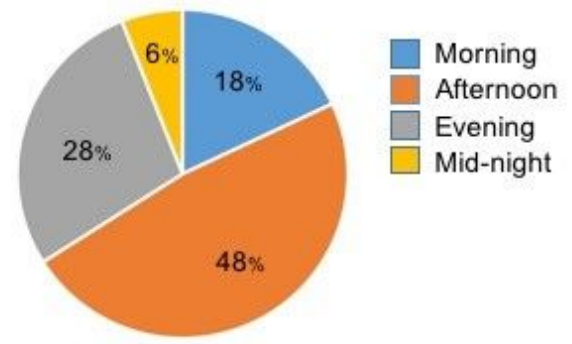

d. How often do you get eyestrain after prolong gameplay

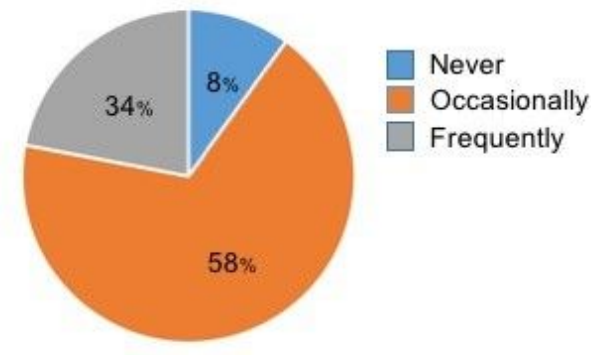

f. How long does it take to have leg numbness in a training/competition

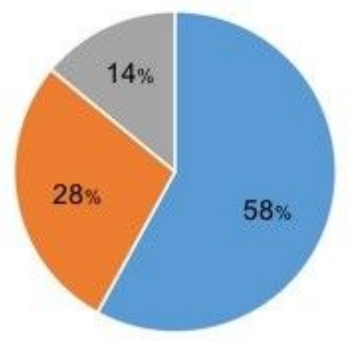

Never

About 4-h training

About 8-h training

\section{Figure 1}

Reported fatigue characteristics in smartphone-game training and competition 


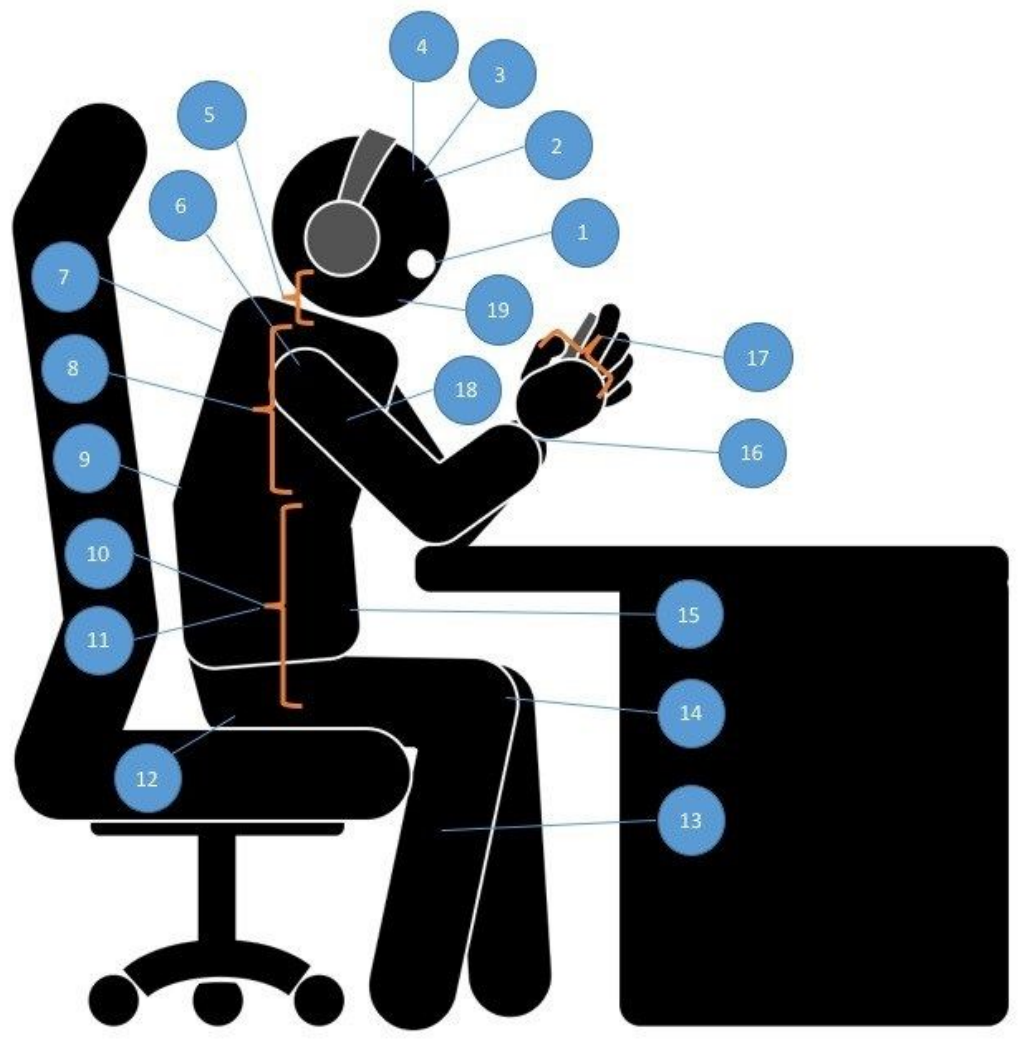

\begin{tabular}{|l|c|}
\hline \multicolumn{1}{|c|}{ Injury/Complaint } & $\mathrm{N}(\%)$ \\
\hline 1. Dry eyes & $9(18 \%)$ \\
\hline 2. Headache / dizziness & $20(40 \%)$ \\
\hline 3. Somnipathy & $3(6 \%)$ \\
\hline 4. Anxiety Neurosis & $3(6 \%)$ \\
\hline 5. Cervical spondylosis & $21(42 \%)$ \\
\hline 6. Shoulder pain & $8(16 \%)$ \\
\hline 7. Pain around the scapula & $12(24 \%)$ \\
\hline 8. Thoracicfacet joint disorder & $7(14 \%)$ \\
\hline 9. Scoliosis & $7(14 \%)$ \\
\hline 10. Lumbar intervertebral disc herniation & $7(14 \%)$ \\
\hline 11. Lumbar muscle strain & $7(14 \%)$ \\
\hline 12. Hemorrhoids & $4(8 \%)$ \\
\hline 13. Deep venous thrombosis & $0(0 \%)$ \\
\hline 14. Patellar tendinosis & $3(6 \%)$ \\
\hline 15. Interogastritis & $11(22 \%)$ \\
\hline 16. Carpal tunnel syndrome & $4(8 \%)$ \\
\hline 17. Finger tenosynovitis & $19(38 \%)$ \\
\hline 18. Angina & $6(12 \%)$ \\
\hline 19. Rhinitis & $16(32 \%)$ \\
\hline
\end{tabular}

Figure 2

Injury location and frequency profile

\section{Image not available with this version}

\section{Figure 3}

This image is not available with this version. 